\title{
A new causal coherence law in quantum physics revealed
}

\author{
Geesink $\mathrm{JH}^{1}$ and Meijer $\mathrm{DKF}^{2 *}$ \\ ${ }^{1}$ Previous Project Leader Nanotechnology, DSM, Geleen, Netherlands \\ ${ }^{2}$ Pharmacokinetics and Drug Targeting, University of Groningen, Groningen, Netherlands
}

A mathematical basis for spectrum of discrete Electromagnetic Field (EMF) frequencies, that were shown to affect health and disease [1], was elaborated and generalized [2]. The particular EMF pattern was earlier revealed by us through a meta-analysis of more than 500 biomedical publications that reported life-sustaining as well as life-decaying EMF frequencies. The detected eigenfrequencies could be arithmetically scaled according to an adapted Pythagorean tuning. The particular semi-harmonic scale exhibits a core pattern of twelve eigenfrequency functions with adjacent self-similar patterns, according to octave hierarchy [1]. The mathematical analysis shows that the derived arithmetical scale exhibits a sequence of unique products of integer powers of 2, 3 and a factor $\sqrt{ } 2$. These discrete eigenfrequency values can be related to supposed bio-resonance of solitons or polaron quasiparticles in life systems. Bio-solitons are conceived as self-reinforcing solitary waves, that are constituting local fields, being involved in intracellular geometric ordering and patterning, as well as in intraand intercellular signaling. Additional literature search, revealed very similar frequency patterns in the color spectrum, for wave resonances of nucleotides in aqueous solution, for a candidate RNA-catalyst, as well as for sound-induced vibrations evoked in thin vibrating membranes as reported by Chladny. This collective evidence points at a generalized biophysical algorithm underlying complexity in nature, evidently manifest in both animate and non-animate modalities, coined by us the Generalized Musical (GM) principle. The particular semi-harmonic frequency spectrum may reflect a discrete pilot-wave structure that can be interpreted as a, so called, hidden variable in Bohm's causal interpretation of quantum field theory and is mathematically expressed as follows:

$$
E_{n}=h \omega_{\text {ref }} 2^{n} 3^{m}\left(2^{p}\right)
$$

In view of the current interest in coherent states and entanglement in quantum biology, we subsequently performed a meta-analysis of 60 papers in physics that deal with the influence of electromagnetic frequencies on the promotion of entangled states in, so called, EPR experiments [3]. Einstein, Podolsky and Rosen originated the EPR-correlation thought experiment for quantumentangled particles, in which particles are supposed to react as one body. The meta-analyses of the EPR-experiments learned that entanglement, achieved in the experiments is real, and that the applied frequencies are located at discrete coherent configurations. Strikingly, all analyzed EPR-data of the independent studies fit precisely in the derived scale of coherent frequency data and turned out to be virtually congruent with the above mentioned semiharmonic EM-scale for living organisms. This implies that the same discrete coherent frequency pattern of EM quantum waves, that determine local and non-local states, is also applicable to biological order and that quantum entanglement is a prerequisite for life. The study may indicate that the implicate order of pilotwave steering system, earlier postulated by David Bohm is composed of discrete entangled EM wave modalities, related to a pervading zero-point energy information field. The GM- biophysical principle was also applied to the areas of cancer therapy [4] and consciousness $[5,6]$.

In a follow-up study, the model was further applied to the data of a meta-analysis of earlier measured discrete energies of 37 different elementary particles, as well as zero-point energies of elements [7]. Further support was found for the GM-model in that it underlies the quantum field theory of subatomic particles, implying that an ontological basis of the Standard Model was found (Figure 1). It was shown that the GM-scale is frequency-locked with zero-point oscillations, and thereby evidently implies involvement of entangled states. The present theory combines quantum mechanics and classical periodic systems, obeys to locality and solves the "hidden variable theory of David Bohm". The pattern of electromagnetic field eigenvalues, within a broad range of discrete frequencies, as revealed in the present studies, points at a de Broglie/Bohm type of causal interpretation of quantum mechanics, implying an integral resonant pilot-wave/particle modality.

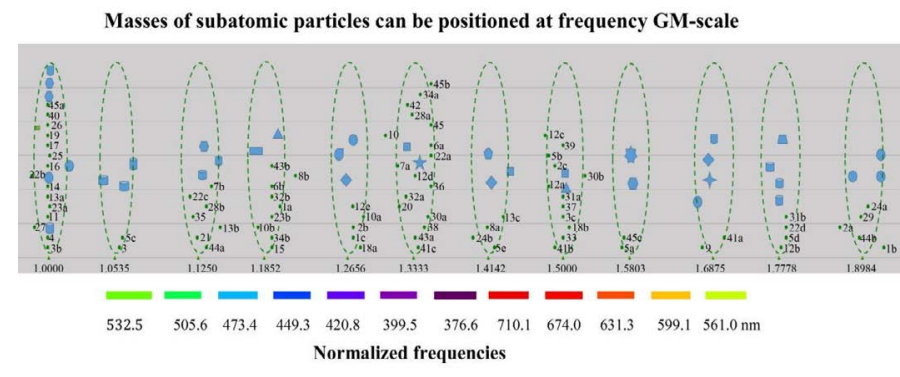

Figure 1. Masses of Subatomic particles can be positioned at frequency GM-scale

\section{References}

1. Geesink JH, Meijer DKF (2017) Electromagnetic Frequency Patterns that are Crucial for Health and Disease Reveal a Generalized Biophysical Principle: the GM scale. Quantum Biosystems 8: 1-16.

2. Geesink JH, Meijer DKF (2018) Mathematical Structure of the GM Life Algorithm that May Reflect Bohm's Implicate Order. J Modern Physics 9: 851-897.

Correspondence to: Meijer DKF, Pharmacokinetics and Drug Targeting, University of Groningen, Groningen, Netherlands, E-mail: meij6076@planet.nl

Received: March 11, 2018; Accepted: March 22, 2018; Published: March 27, 2018 
3. Geesink JH, Meijer DKF (2018) A harmonic-like electromagnetic frequency pattern organizes non-local states and quantum entanglement in both EPR studies and life systems. J Modern Physics 9: 898-924.

4. Meijer DKF, Geesink JH (2018) Favourable and Unfavourable EMF Frequency Patterns in Cancer: Perspectives for Improved Therapy and Prevention. $J$ Cancer Therapy 9: 188-230.
5. Meijer DKF, Geesink JH (2016) Phonon Guided Biology. Architecture of Life and Conscious Perception are mediated by Toroidal Coupling of Phonon, Photon and Electron Information Fluxes at Discrete Eigenfrequencies. Neuro Quantology 14: 718-755.

6. Meijer DKF, Geesink JH (2017) Consciousness in the Universe is Scale Invariant and Implies the Event horizon of the Human Brain. Neuro Quantology 15: 41-79.

7. Geesink JH, Meijer DKF (2018). Semi-Harmonic Scaling enables Calculation of Masses of Elementary Particles of the Standard Model. J Modern Physics 9: 925-947.

Copyright: $\odot 2018$ Geesink HJ. This is an open-access article distributed under the terms of the Creative Commons Attribution License, which permits unrestricted use, distribution, and reproduction in any medium, provided the original author and source are credited. 